\title{
Las sustancias inteligibles y su operación en el Comentario al Libro de las causas de Tomás de Aquino
}

\author{
VICTORIA ARROCHE
}

Universidad de Buenos Aires

Resumen: En este artículo intentaré explorar cuatro puntos relacionados con el Comentario de Tomás de Aquino al Libro de las causas. El primero, vinculado a la combinación de dos perspectivas metafisicas: a) la tradición filosófica neoplatónica y b) las religiones monoteístas y creacionistas que se desarrollaron en el mundo islámico y el cristiano. El segundo, relacionado con el modo en que el comentario de Aquino aglutina y fusiona temas centrales de la metafísica procleana. En ese sentido, en tercer lugar, analizaré el uso argumentativo que hace Tomás de la cuestión de la esencia y la operación de las sustancias inteligibles. Por último, intentaré presentar una conclusión teniendo en cuenta las consecuencias de esa argumentación, en relación con el carácter autosubsistente del alma.

Palabras clave: metafísica, Proclo, Tomás de Aquino, inteligencias, almas. 


\title{
Intellegible Substances and their Operation according to Thomas Aquinas' Commentary on the Book of Causes
}

\begin{abstract}
In this article, I will examine four issues related to Thomas Aquinas' Commentary on the Book of Causes. The first, refers to the combination of two metaphysical perspectives: a) the Neoplatonic philosophical tradition and b) the monotheistic and creationist religions developed in the Islamic and Christian world. The second, is connected to the way in which Aquinas collects and amalgamates central themes of proclean metaphysics in his commentary. In this sense, thirdly, I will analyze Thomas' use of the essence and the operation in intelligible substances theory. Finally, I will try to conclude, showing the scope of his argumentation in relation with the self-subsistent character of the soul.
\end{abstract}

Key-words: metaphysics, Proclus, Thomas Aquinas, intelligences, souls.

\section{Platonismo, Aristotelismo, Cristianismo: el contexto históvico-cultural de la vida de Proclo}

—1 contexto histórico, político y social en el que transcurrió la vida

Ede Proclo (411-485) suele interpretarse como una transición. Esta idea parece indicar un modo de comprender aquellos períodos de la historia que se caracterizan por la imprecisión o dificultad para definir sus límites. A su vez, esta situación particular, determinaría la producción intelectual de quienes desarrollaron su pensamiento en ese momento.

Uno de los motivos por los que esas "transiciones" entre épocas históricas promueven cierto tipo de discusiones y explicaciones es que consisten, ciertamente, en períodos en los que conviven diversas culturas y tradiciones filosóficas. Parece que también es posible comprender el pensamiento de aquellos que vivieron transiciones históricas, simultáneamente como precursores y continuadores de movimientos de ideas, es decir, filósofos que ofrecen una síntesis de tradiciones anteriores y, al mismo tiempo, habilitan con sus reflexiones el tránsito hacia un nuevo marco teórico o paradigma. Así pues, resulta interesante tener en cuenta que Proclo vivió y elaboró su obra en períodos signados por profundas transformaciones socio-políticas que se revelan, quizás, en la profusión y convivencia de diversas culturas e ideas. En ese sentido, el contexto cultural del siglo V, puede caracterizarse como un "encuentro" entre neoplatonismo y cristianismo (D’Amico 2008: 13-28). 
Desde un punto de vista histórico-político, los cambios y movimientos acaecidos en el siglo $\mathrm{V}$ se relacionan con algunos acontecimientos determinantes como la devastación de las fronteras del Imperio Romano Occidental (406-410), las grandes migraciones bárbaras, la invasión de los Hunos (440452), el saqueo de Roma por parte de losVisigodos (410) y los Vándalos (455). En lo que atañe al aspecto más bien cultural, la categoría de "transición", suele también aplicarse a este momento histórico para describirlo como un lento pasaje del paganismo al cristianismo (Siorvanes 1996: 6-7).

Por otra parte, resulta manifiesto que una de las características sobresalientes de la filosofia neoplatónica es que se presenta como una elaboración llevada a cabo por pensadores que se consideraron a sí mismos discípulos de Platón y, en ese marco teórico, sintetizaron elementos provenientes, en gran medida, del aristotelismo y del estoicismo. Es conocida la complejidad que conlleva caracterizar a la primera de estas tradiciones, así como también la dificultad para describir la fusión ulterior de esas ideas neoplatónicas con nociones propias del cristianismo. Con todo, la dinámica de ese "encuentro" permite dar cuenta del surgimiento de un tipo de pensamiento al que puede denominarse "neoplatonismo medieval" que, en el Occidente latino, adquirió una fisonomía propia.

Algunos de los filósofos que representaron esta línea de pensamiento resultaron fundamentales para el desarrollo de la metafísica en la Edad Media (Gersh 2014:125-132). En especial, respecto de Proclo, existen al menos dos aspectos que nos permiten caracterizar la recepción de su obra en el Occidente latino como un acontecimiento peculiar. En primer lugar, durante la Edad Media, su pensamiento fue conocido no solamente en el mundo latino sino también en el islámico. En segundo lugar, sus ideas circularon en esos ámbitos intelectuales, o bien de forma anónima o bien a través de otros autores que modificaron y expusieron su filosofia pero sin explicitar el nombre de Proclo. Por ello, puede afirmarse que la recepción medieval de sus ideas tuvo lugar de modo directo e indirecto. ${ }^{1}$ Justamente, en lo que atañe a la figura de nuestro autor y a la interconexión entre neoplatonismo y cristianismo, los pensadores latinos medievales tuvieron contacto, ya desde el sigloVI, con algunas categorías fundamentales del pensamiento de Proclo a través de la lectura del Pseudo Dionisio (circa 460-530) (Ludueña 2008: 75 y ss.; Siorvanes 1996: 31 y ss.; Dillon 2014: 111 y ss.). En efecto, el corpus

\footnotetext{
${ }^{1}$ Se denomina "recepción indirecta" a las paráfrasis y modificaciones de la obra original de Proclo que circularon tanto en Oriente como en Occidente, en general, con el nombre de otro autor. La vía directa consiste en las traducciones realizadas a partir de las obras originales en griego.
} 
Dionysiacum fue una de las vías indirectas de recepción del sistema filosófico procleano en Occidente y su autoridad fue largamente aceptada por los pensadores medievales en virtud de la leyenda, construida por el propio Dionisio, de su conversión al cristianismo durante el discurso de San Pablo en el Areópago. Por otra parte, en el mundo islámico, el filósofo neoplatónico fue estudiado en profundidad aunque su influencia en la formación de la filosofia árabe se ejerció con el nombre de Aristóteles (D’Ancona 2014: 137 y ss.). En efecto, en el siglo IX, una compilación de las Enéadas de Plotino y de los Elementos de Teología de Proclo circuló en el ambiente árabe adjudicándose su autoría al Estagirita. Esa compilación, una vez traducida al latín, tuvo gran fortuna en Occidente con el nombre Liber de causis (Libro de las causas). Precisamente, este tratado fue conocido en el siglo XII como un texto aristotélico y fue estudiado a modo de complemento de la Metafísica de Aristóteles como la culminación teórica del libro Lambda de esa obra (Dodds 1963: 24-32).

En cuanto a la recepción directa de Proclo, la primera noticia que registra Occidente es también en el siglo XII cuando los intelectuales medievales dispusieron de la Elementatio physica, traducida en el círculo de Aristipo de Catania (Imbach 1978: 431). Pero el acceso a las obras originales de Proclo se intensificó y resultó de mucha importancia durante el siglo XIII, cuando el dominico Guillermo de Moerbeke realizó traducciones de algunos de sus textos del griego al latín. En efecto, entre ellos se encuentra una traducción parcial del Comentario al Timeo (1264) que parece haber completado luego, durante su permanencia en Corinto (1283-1284). En 1268 tradujo los Elementos de teología, en 1280 los Tria opuscula y en 1286 el Comentario al Parménides (Steel 2014: 247-263; Klibansky 1943: 284.).

En este trabajo nos interesa destacar especialmente la primera de esas obras, Elementos de teología, pues es aquella con la que contaba Aquino al momento de escribir su Comentario al Libro de las causas. ${ }^{2}$ Precisamente, en 1272 al componer ese texto, Tomás conectó, por primera vez durante la Edad Media, el tratado anónimo con su fuente procleana y con la obra de Dionisio Areopagita y, de esa forma, delimitó una parte importante de lo que denominamos "filosofía neoplatónica medieval". Así, quizás paradójicamente, los límites conceptuales de la tradición neoplatónica parecen haberse establecido, en el siglo XIII, en un marco teórico que posteriormente, algunas corrientes historiográficas -vigentes hasta bien entrado el siglo XX- han denominado "pensamiento aristotélico-tomista".

${ }^{2}$ Tomás de Aquino, Expositio Super librum De causis que citaremos de la siguiente forma: Expositio y el número de proposición (lectio) correspondiente. 


\section{La influencia indirecta de Proclo a través del Libro de las causas}

Tos historiadores de la filosofia medieval han fortalecido la idea de que "la historia de la recepción del saber" y "el encuentro entre culturas" pueden considerarse fenómenos esencialmente medievales (Florido 2005: 2). Ello se debe a que, cada vez más, los medievalistas ponen en evidencia la influencia que tuvo en la formación de la cultura occidental, la trasmisión del saber científico, literario y filosófico desde Oriente a Occidente. El fenómeno denominado traslatio studiorum se presenta, de ese modo, como un complejo proceso de trasmisión del pensamiento filosófico desde Grecia, Bizancio, Egipto o Arabia a Occidente. Al mismo tiempo, señala la transformación de ese pensamiento en un "nuevo saber" que, amparándose en el nombre de Aristóteles como símbolo de su carácter filosófico ofreció, paulatinamente, tanto en Oriente como en Occidente, explicaciones científicas o metafísicas que habían sido patrimonio casi exclusivo del saber teológico. El Libro de las causas constituye un ejemplo de esa trasmisión pues es una obra cuyo contenido doctrinal remite al pensamiento de Proclo y de Plotino pero fue conocida como un texto aristotélico. Se trata, precisamente, de un tratado anónimo, ampliamente difundido en el Occidente latino a partir del siglo XII. En cuanto a su estructura, se compone de treinta y una proposiciones ${ }^{3}$ que sintetizan algunas de las tesis más significativas de los Elementos de Teología (que en la obra de Proclo se desplegaban en doscientos once proposiciones) e incorporan algunas nociones provenientes de la llamada "plotiniana arábica", esto es, un conjunto de textos extraídos de las Enéadas de Plotino, traducidos al árabe y utilizados en la producción de diversos textos (D’Ancona 1986: 42-47; De Libera 1990: 347-378). Precisamente, una de las modificaciones más notables respecto de la fuente procleana es la organización de lo real según tres hipóstasis: la Causa Primera (llamada también Bien, Uno Puro o Dios), la Inteligencia y el Alma. El planteo de los Elementos -sumamente complejo en virtud de la postulación de mediaciones y relaciones que garantizan la continuidad del sistema- fue reemplazado, en el De causis, por un esquema mucho más reducido en el que el ámbito inteligible

${ }^{3}$ El Líber de causis, en su versión árabe, consta de 31 proposiciones. Los números entre paréntesis corresponden a las proposiciones que comenta Tomás de Aquino, ya que a partir del desdoblamiento de la proposición 4 el tratado presenta 32 proposiciones. Las citas textuales estarán referenciadas con las iniciales del texto $L d C$ y ambos números de proposición al lado. 
se extiende en esas tres instancias que recrean, nominalmente, el orden plotiniano de la realidad. ${ }^{4}$

El Libro de las causas circuló bajo el nombre de Aristóteles tanto en el ámbito intelectual islámico cuanto en el Occidente latino hasta que, como mencionamos, Tomás de Aquino reveló su fuente directa en su comentario. Hasta entonces, los intelectuales universitarios medievales consideraron al texto un estudio complementario de los conocimientos que el Estagirita ofrecía en su Metafísica. Según lo establecido en la Guía del Estudiante de 1255 se juzgó al tratado anónimo como una continuación del Libro XII del texto aristotélico. Justamente, en la Guía ambas obras -Libro de las causas y Metafísica - resultan lectura obligatoria para acceder a la Facultad de Artes de la Universidad de París (D’Ancona 2005: 818 y ss; Bertelloni 2001: 197).

En un trabajo fundacional sobre el origen y el contenido metafísico del De causis, Saffrey (1963) puso de manifiesto los problemas relativos a la identificación de su autor. Si bien ese tema fue objeto de profundos debates es importante señalar que, actualmente, parece existir consenso entre los intérpretes en considerar que el texto fue escrito por al-Kindi o por alguno de sus discípulos en ámbito islámico en Bagdad, entre los siglos IX y X y luego traducido al latín entre 1167 y 1184, en Toledo, por Gerardo de Cremona (D’Ancona 2005: 811 y 1995: 155-194; De Libera 1990: 350). En ese sentido, nos interesa subrayar que la tesis que sostiene el origen árabe del texto puede conciliarse con su estudio en el marco de la doctrina metafísica aristotélica porque, verdaderamente, el fenómeno de asimilación de doctrinas neoplatónicas y su atribución a Aristóteles en la esfera intelectual islámica, no está representado solo por la obra que nos ocupa. En efecto, otros de los escritos que comparten esas mismas cualidades son la Pseudo-Teología de Aristóteles, la Carta sobre la ciencia divina y los Dichos del sabio griego. Estos tres escritos junto con el Libro de las causas, que pertenecen a la tradición neoplatónica por su contenido filosófico, comparten al menos dos características. En primer lugar, se trata de textos elaborados sobre la base de una modificación o paráfrasis de obras pertenecientes, por ejemplo, a Plotino, Proclo o Porfirio y cuya autoría se adjudicó a otros filósofos, en particular a Aristóteles (D’Ancona 2003: 72-91). En segundo lugar, en estas obras se verifica la cancelación o modificación de aquellas tesis incompatibles con el monoteísmo. En este sentido, el tratado anónimo es un ejemplo de este tipo de trasmisión en la que un texto de

${ }^{4}$ Junto con esta adopción se eliminan los dioses de Proclo. Sobre las tres hipóstasis del Liber y su vinculación con la filosofía de Plotino cfr. D’Ancona 1995: 46. 
origen neoplatónico se atribuye a Aristóteles y en el que se combinan dos tradiciones filosóficas -la neoplatónica y la aristotélica- en el marco de un pensamiento monoteísta (D’Ancona 1986: 43-45). Asimismo, en estas producciones filosóficas originalmente árabes, abundan y hasta predominan temas específicos del neoplatonismo como, por ejemplo, la teoría de la emanación, la estructura jerárquica del cosmos, la doctrina de las inteligencias separadas, la procesión y el retorno de las inteligencias y las almas (D’Ancona 2003: 75-77 y 1986: 44).

A pesar de su origen árabe, lo cierto es que el Libro de las causas tuvo mucha mayor fortuna en el ámbito filosófico del Occidente latino que en el islámico. La incorporación definitiva de los textos aristotélicos a la Facultad de Artes de la Universidad de París en 1255 marca a la metafísica como la parte culminante de la filosofía, como la ciencia de las realidades separadas de materia. Esta asignatura se estudiaba según su articulación en tres libros principales: la metafísica vetus, fórmula con la que se denominaba a la traducción greco-latina de Giacomo Veneto (1128-1155) o a la revisión anónima y parcial de esa traducción (D’Ancona 2005: 819-820). El segundo texto obligatorio era la metafísica nova que habría correspondido a la nueva traducción del árabe al latín. Ambas traducciones fueron utilizadas para reconstruir la Metafísica de Aristóteles, es decir, completar las lagunas y la ausencia de los Libros 1-4 de la versión árabe. Por último, el Libro de las causas que constituía una obra de lectura obligatoria. La reconstrucción del tratado aristotélico finalizaba en el libro Lambda que aparecía con el número XI. En ese Libro, los intelectuales medievales leyeron una doctrina que trataba sobre la causalidad universal del Primer Motor Inmóvil y la conectaron con la teoría causal expuesta por "Aristóteles" en el Libro de las causas. El texto anónimo se convirtió, de ese modo, en "la tercera parte de la ciencia de las cosas totalmente separadas de la materia y del movimiento, según el ser y la definición” (D’Ancona 2005: 821, n.163).

\section{Elementatio theologica $y$ Liber de causis en la Expositio Super Librum de causis de Tomás de Aquino}

Ta proposición 14 (15) del Libro de las causas describe el retorno

_completo (reditio completa) de una hipóstasis específica, a saber, la inteligencia. La fórmula expresa la capacidad de esa sustancia para retornar hacia su esencia.

El minucioso trabajo filológico y filosófico de Tomás de Aquino en su Expositio super librum De causis, nos otorga la oportunidad de acceder a las proposiciones de la Elementatio theologica que constituyen las fuentes del 
tratado anónimo. En su comentario a la proposición 15 de ese tratado, ${ }^{5}$ Aquino hace referencia a las proposiciones $15,16,43,44,83$ y 186 de la obra de Proclo. Las dos primeras se encuentran entre los enunciados con los que el autor neoplatónico describe la estructura de la realidad. En efecto, Proclo presenta allí una serie de notas que acompañan a los principios automovidos: o bien son incorpóreos o pueden separarse del cuerpo y poseen la capacidad de retornar sobre sí mismos (cfr. Prop. 17). Además, señala los momentos necesarios para que se produzca el despliegue de la realidad, es decir, explica por qué el efecto es otro respecto de su causa y, al mismo tiempo, está esencialmente vinculado a ella. Justamente, se trata de la permanencia (moné/ manens), la procesión (próodos/processio) y el retorno (epistrophé/conversio), instancias que indican no solamente el dinamismo de la realidad sino también la constitución ontológica de las entidades que conforman ese sistema. Por ello, puesto que la fórmula reditio completa expresada en la proposición 14 (15) del Libro de las causas remite al concepto procleano de retorno, también alude a la naturaleza o complexión de las sustancias.

Ahora bien, el autor de los Elementos de teología postula la existencia de ciertas sustancias que pueden causarse a sí mismas, es decir, que al efectuar el movimiento de retorno hacia sí mismas, en rigor, no se convierten hacia una causa externa sino que vuelven hacia su propia esencia y, por eso, su constitución es, ciertamente, una autoconstitución. Proclo denomina a esos principios authypóstaton ${ }^{6}$ y, precisamente, resultan fundamentales para su sistema pues se trata de entidades autosubsistentes y autosuficientes (cfr. Prop. 40). La descripción de estas sustancias se desarrolla desde la proposición 42 hasta la 48.

Asimismo, una realidad que se autoconstituye tiene la capacidad para autoconocerse. Justamente, las entidades denominadas authypóstaton conocen su esencia y, por lo tanto, ejercen una actividad intelectual ya sea por participación o esencialmente. El primer caso, i.e. por participación, lo representan las almas y el segundo, i.e. por esencia, es propio de las sustancias inteligibles (es decir, que pueden ser inteligidas) e inteligentes (es decir, cuya capacidad es intelectual). Las inteligencias autosubsistentes retornan hacia sí mismas y su operación propia consiste en autoconocerse, pues son, simultáneamente, el sujeto que conoce y el objeto conocido.

En ese sentido, la proposición 14 (15) del Libro de las causas asume algunos de estos tópicos procleanos, por ejemplo, la idea de que los cuerpos

\footnotetext{
${ }^{5}$ El número de las proposiciones del Libro de las causas que está entre paréntesis, corresponde al número de proposición que será comentada por Tomás de Aquino en su Expositio.

${ }^{6}$ Este término es transliterado así en la traducción de Moerbeke. Sobre la terminología para traducir authypóstaton, cfr. Gersh 2009: 67, n. 111; Dodds 1963: 43.
} 
no tienen la capacidad para retornar sobre sí mismos, así como tampoco las sustancias divisibles. El autor del De causis, por eso, considera que la inteligencia tiene esa capacidad ya que es incorpórea e indivisible ( $c f r$ r. LdC prop. 6 (7)). Además, según expresa el tratado, la operación propia de la inteligencia es intelectual ( $c f r$ r. $L d C$ prop. 3). Luego, también se conoce a sí misma pues es el sujeto cognoscente y el objeto conocido simultáneamente.

Así, la proposición 14 (15) del Liber de causis expresa:

Todo cognoscente que conoce su esencia retorna a su esencia con un retorno completo. Es así ya que el conocimiento no es sino una acción intelectual. En consecuencia, cuando el cognoscente conoce su esencia, entonces retorna por su operación intelectual a su esencia. Esto no es así, sino porque el cognoscente y lo conocido son una cosa una, porque el conocimiento del que conoce su esencia es desde él y para él: es desde él porque es cognoscente, es para él ya que es conocido. Esto es porque en tanto que el conocimiento es conocimiento del cognoscente y el cognoscente conoce su esencia, su operación es de retorno a su esencia; por lo tanto, su substancia es la que retorna a su esencia otra vez.Y no significo por "retorno de la substancia a su esencia" sino que es estable, fijada por sí misma, no necesitada en su fijeza y en su esencia de ninguna otra cosa que la rija, ya que es substancia simple, suficiente por sí misma ( $L d C$, prop. 14 (15)).

Sin duda, el Comentario de Tomás de Aquino al Libro de las causas merece ser investigado por tratarse del primer texto medieval en el que se identificó el origen neoplatónico del tratado anónimo. Además, resulta especialmente interesante profundizar sobre un posible giro hermenéutico por parte de Aquino, al comentar un texto conocido y estudiado desde el inicio de su carrera pero ahora, disponiendo de la reciente traducción de los Elementos de teología. Asimismo, se ha puesto de manifiesto la gran cantidad de estudios dedicados a la apropiación de temas pertenecientes a la metafísica árabe por parte del Doctor Angélico. En ese sentido, el Libro de las causas fue un recurso de consulta frecuente. Por último, una nueva y aún poco explorada línea de estudios viene concentrándose sobre la influencia del Liber de causis en la teoría gnoseológica del Aquinate (Scarpelli Cory 2017: 185, n. 2).

Mi interés en el comentario de Tomás al De causis y, en particular, a las proposiciones 15 y 25, se vincula con cuatro puntos principales: el primero, concierne al hecho de que en este escrito aparece con claridad la combinación de dos perspectivas metafísicas, esto es, por un lado la tradición filosófica neoplatónica y, por el otro, las religiones monoteístas y creacionistas que se desarrollaron en el mundo islámico y el cristiano. Específicamente, 
este encuentro obliga a reflexionar sobre el concepto de creación entendido como la dación del ser por parte de una causa primera a todas las entidades existentes. El propio Tomás, como veremos, parece ser consciente de este problema. Como consecuencia de esa primera cuestión, se sigue el segundo punto pues, justamente, el comentario de Aquino a estas dos proposiciones aglutina y fusiona temas centrales de la metafísica procleana presentes en los Elementos de teología. En tercer lugar, en el comentario a estas proposiciones, el Doctor Angélico trata específicamente sobre la sustancia y la operación de las inteligencias para mostrar, por una parte, que la esencia es superior a la actividad y, por otra parte, para utilizar ese principio en su argumentación acerca de la diferencia metafísica entre las nociones de creación y generación de una sustancia. Por último, a partir de este análisis, intentaré precisar el alcance que pueda tener la argumentación de Aquino respecto del alma ya que, al igual que la inteligencia, se trata de una sustancia autosubsistente.

$\mathrm{Al}$ inicio de su comentario a la proposición 15 del Liber de causis, Tomás de Aquino cita la proposición 15 de los Elementos de teología tal como se lee en la traducción de Moerbeke: "todo lo que puede volver hacia sí mismo es incorpóreo". Y luego, reconfirma esa premisa con un fragmento de la proposición 16 de Proclo: "todo lo que puede volver hacia sí mismo posee una sustancia separada de cualquier cuerpo". Sobre este punto, además, Aquino explica la prueba que incluye la proposición 16 del texto de Proclo. Dice el Doctor Angélico:

Puesto que el cuerpo es incapaz de volver hacia sí mismo, como es evidente a partir de la premisa de la proposición que ya se tenía [la 15 de los Elementos], se sigue que "volver hacia sí mismo" sea una operación separada del cuerpo. Pero, si esta operación es separable del cuerpo, es necesario que también la sustancia sea separable. De ahí que todo lo que puede volver hacia sí mismo es separable del cuerpo (Expositio, lectio 15).

En el sistema procleano, las características enumeradas en la serie de proposiciones que van de la 14 a la 20 de los Elementos de teología permiten establecer una escala de realidades desde lo superior hacia lo inferior. En el nivel más alto de lo real se encuentra lo Uno y luego, por participación, la realidad se despliega en el plano de la Inteligencia, sigue el ámbito del Alma y finaliza con el del Cuerpo. Como mencionamos, entre las realidades superiores se encuentran las authypóstata, es decir, aquellas entidades que son causa de sí mismas (Dodds 1963: 224-225) y, por ello, subsisten sin la ayuda de un soporte material como el cuerpo. Por último, como muestra Aquino en su Expositio al citar la proposición 16 de los Elementos de teología, en la escala metafisica establecida por Proclo, la esencia es superior a la actividad 
de una sustancia. Esta última aseveración resulta de gran relevancia pues el lema "la operación sigue a la esencia" (operatio sequitur esse) ${ }^{7}$ es un verdadero principio metafísico que determinó una concepción de la realidad compartida por muchos intelectuales medievales.

Como dijimos, Aquino también identifica como fuentes de la proposición 15 del Libro de las causas, a las proposiciones 43 y 44 de los Elementos. Estos dos enunciados son particularmente importantes, porque aquí Proclo afirma que las sustancias que se autonconstituyen son causas de sí mismas y se dan el ser a sí mismas.

Tomás lo explica de la siguiente forma:

Todo lo que es capaz de tornar sobre sí mismo es authypóstaton, esto es, subsistente por sí.Y esto se prueba porque cada realidad se vuelve a aquello por lo cual es. Por lo tanto, si algo se vuelve sobre sí mismo según su ser, es necesario que subsista en sí mismo (Expositio, lectio 15).

En la proposición 44 de Proclo, según Tomás, se afirma la superioridad de la sustancia sobre la operación. Esa proposición expresa que "toda realidad que es capaz de volver sobre sí misma según su operación, también es capaz de volver según su sustancia" (lectio 15). En efecto, según Aquino, sobre la base del principio que postula la superioridad de la sustancia respecto de la operación, Proclo había establecido, en la proposición 16, la necesidad de que una sustancia sea separable del cuerpo para poder retornar sobre sí misma. Ahora, en la proposición 44 de los Elementos de teología, ese principio metafísico funcionaría para mostrar que si una sustancia es capaz de volver sobre sí misma (secundum operationem) con más propiedad entonces podrá subsistir por sí misma (secundum substantiam), es decir, posee la facultad de darse el ser a sí misma. En efecto, si así no fuera "se seguiría que la operación sería mejor y más perfecta que la sustancia" (lectio 15).

Ahora bien, uno de los problemas más interesantes que surgen al abordar el análisis del Libro de las causas radica en definir el modo en que su autor resuelve el modelo metafisico presente en los Elementos de teología, teniendo en cuenta que el concepto de "participación", que resultaba esencial en el texto de Proclo, está completamente ausente en el tratado anónimo. En ese sentido, las nociones de "participación" y de "semejanza" en la filosofia procleana indicaban un supuesto ontológico fundamental, a saber, que la complicación del efecto con su causa es condición necesaria para que el

\footnotetext{
${ }^{7}$ Tomás de Aquino, Suma Teológica I-II, q. 4, art. 5.
} 
efecto sea determinado como tal, es decir, que el efecto se constituya como uno-otro de su causa (cfr. Prop. 1-6). Asimismo, al exponer su teoría acerca de las sustancias autoconstituyentes, Proclo afirmaba que su operación (el retorno hacia sí mismas), que es connatural a su esencia, se desarrollaba con autonomía de una causa externa.

Tomás de Aquino nos ofrece una interesante clave interpretativa sobre este punto que intentaremos examinar con cierto detenimiento. En efecto, al inicio de su Comentario señala que el autor del Libro de las causas utiliza el "verbo influir y Proclo usa el verbo producir que expresa la causalidad de la causa eficiente" (lectio 1). ${ }^{8}$ La interpretación de Aquino nos enfrenta a la idea de que la noción de influencia, que aparece en la primera proposición del De causis, puede equipararse al concepto de producción al que recurre Proclo para describir el vínculo de participación entre la causa y el efecto.

En cuanto a la noción de "causalidad eficiente", en rigor, el tratado anónimo afirma explícitamente que existen dos modos de causar: el primero, vinculado con la operación que efectúa la causa primera y, el segundo, relativo a la operación de la inteligencia y del alma. Según el Liber, la causa primera ejerce su influencia por el modo de la creación (modus creationis) y las causas segundas, por el modo de la formación (modus formae). Esta distinción entre estos dos modos de causar, que aparece en la proposición 18, no está presente en los Elementos de teología de Proclo. En efecto, en esa obra, la forma inmanente en el efecto (in-existit) vinculaba -por semejanza- a este con su causa y ello constituía, precisamente, la participación (procesión y retorno). En el tratado anónimo, en cambio, la influencia es una operación ejercida por la causa primera que otorga y conserva al efecto en el ser. Justamente, allí se dice que la causa primera "da a todas las cosas el ente [...] por el modo de creación” (LdC Prop. 17 (18)), mientras que las causas segundas imprimen una forma que especifica al ente creado. Así, la influencia de la Causa Primera consiste en transmitir una primera perfección a sus efectos. Esa perfección es el ser y su trasmisión se efectúa por el modo de la creación.

Asimismo, desde el inicio del Libro de las causas, en la proposición 1, se establece un principio metafísico que rige todo el contenido de la obra y puede expresarse así: la causa primera constituye el fundamento ontológico de las causas segundas y de sus efectos. Ello se expresa caracterizándola como creadora de toda la realidad. De ese modo, las causas segundas obtienen el ser (esse) y la operación (virtus) de la causa primera y se conservan en el ser gracias a su potencia.

\footnotetext{
${ }^{8}$ Sobre la noción de creación en Tomás de Aquino, Aertsen 1988:120-122; Acar 2005:197199.
} 
El modelo creacionista y monoteísta al que pertenece el Libro de las causas, por lo tanto, no postula la existencia de sustancias autoconstituyentes, esto es, que se causan a sí mismas o cuyo ser depende de sí mismas. En cambio, en cuanto a la actividad, esas realidades poseen una virtus propia, es decir, que depende de ellas mismas, si bien ello no impide que la potencia de la causa primera continúe ejerciéndose siempre junto con la de las causas segundas. Precisamente, la causa primera mantiene a todos los efectos en el ser pues su influencia es una operación permanente y más poderosa que la de las causas segundas.

Ahora bien, puesto que, según el principio establecido en los Elementos de teología, la esencia es superior a la actividad, si el autor del Libro de las causas sostiene, en la proposición 14 (15), que la inteligencia realiza un retorno completo sobre sí misma, cabe suponer que, cuando expresa que toda sustancia intelectual es "subsistente y estable por sí misma" refiere a las características de una sustancia autoconstituyente que, respecto de su ser, no depende de una causa externa. Sin embargo, como Tomás de Aquino señala en su comentario a la proposición 25 , con relación a las inteligencias:

Nadie malinterprete que las sustancias de esta clase no tendrían causa de su ser, puesto que anteriormente se ha dicho que "todas las realidades tienen esencia por el ente primero" (Expositio, lectio 25).

Así, Aquino pone en evidencia la existencia de un cambio conceptual, que el autor del Liber materializó al insertar las proposiciones procleanas en el contexto de una metafisica creacionista y monoteísta. El Doctor Angélico demuestra, de ese modo, su honestidad intelectual ya que, lejos de esconder las fuentes del tratado anónimo, las expone tal como Moerbeke las tradujo, esto es, apegado a la letra del texto de Proclo. (Scarpelli Cory 2017: 212). Pero, asimismo, exhibe también su interés por encuadrar estas reflexiones metafisicas que pertenecen a la ciencia divina, en un marco no dogmático. En efecto, como veremos, Aquino propone una explicación sobre el punto en cuestión. El comentario a la proposición 25 de su Expositio dice:

Son subsistentes por su propia esencia aquellas sustancias que son solamente formas, no basadas en la materia; y, de esta manera, es imposible que sean generadas. Por otro lado, hay que tener en cuenta que la primera proposición es una conclusión de esta segunda; pues anteriormente se ha probado [proposición 15] que todas las sustancias inteligentes son subsistentes por su propia esencia. Así pues, si "toda sustancia subsistente por su propia esencia no es generada", se sigue que toda sustancia inteligente no es generada (Expositio, lectio 25). 
La relación de consecuencia propuesta por Tomás, entonces, puede esquematizarse con el siguiente silogismo:

1. Toda sustancia inteligente es subsistente por su propia esencia. ${ }^{9}$

(Omnes substantiae intelligentes sunt stantes per essentiam suam)

2. Toda sustancia que subsiste por su propia esencia es no generada. (Omnis substantia stans per essentiam suam est non generata)

3. Por lo tanto, toda sustancia inteligente es no generada. (Omnis substantia intellectualis sit non generata)

Según explica Aquino, cuando el Liber de causis dice que las sustancias inteligentes no son generadas por otro, está suponiendo. en primer lugar, que tales entidades son, por naturaleza, capaces de intelección. En segundo lugar, también señala que se trata de sustancias simples, es decir, que no están compuestas de materia y forma como otro tipo de realidades. Por último, su inmaterialidad indica que son intelectuales en acto. Precisamente, la ausencia de materia comporta la ausencia de potencialidad y, por lo tanto, su ser es en acto (esencia) y también su operación, que consiste en ejercer su capacidad intelectual continuamente (D’Ancona 1986: 393, n. 7).

A partir de estas características propias de las sustancias inteligentes separadas de materia podemos obtener una definición del concepto de "generación". Tomás señala, en su comentario a la proposición 25:

En efecto, es evidente que toda cosa generada es, de por sí imperfecta, porque es un ente en potencia y, por eso, requiere ser completado o perfeccionado, por aquello a partir de lo cual es generado, es decir, por el generante que conduce a ese ente de la potencia al acto.Y un indicio de esto se ve en el hecho de que la generación no es otra cosa que un cierto camino que va de lo incompleto al completo opuesto (Expositio, lectio 25. Cursiva en el original).

Justamente, las sustancias inteligentes que se caracterizan por ser no generadas, no necesitan cumplir con el pasaje de lo imperfecto a lo perfecto descripto en el texto anterior:

$\mathrm{Si}$, por lo tanto, existe algo que no requiere de otro para formarse, sino que, eso mismo es la causa de su propia formación entonces, puesto que la sustancia es su forma, se sigue que una entidad así es siempre completa o perfecta.Y así, en ella no puede haber un pasaje de lo imperfecto a lo perfecto sino que continuamente y por sí misma es ente y uno como se dice en el libro VIII de la Metafísica (Expositio, lectio 25. Cursiva en el original).

\footnotetext{
${ }^{9}$ Cfr. Expositio, lectio 15.
} 
Ahora bien,Tomás explica que en todas las sustancias se verifica que la esencia es, en sentido primario, su forma (cfr. lectio 25). Pero la generación de las entidades cuya forma se cimienta en la materia, se define como el pasaje de la potencia al acto y, por lo tanto, de la imperfección a la perfección. Esto significa, entonces, que las sustancias compuestas por materia y forma son generadas por una causa externa a ellas, a saber, una causa agente que actualiza lo que está en potencia. En este caso, según Aquino, la proposición 25 hace referencia al modo en que actúa esa causa agente o eficiente.

Sin embargo, existen otro tipo de sustancias que, a diferencia de las anteriores "son solamente formas, no insertas en la materia y que, por lo tanto, subsisten por su esencia [y] es imposible que sean generadas" (lectio 25). No obstante, eso no significa que este tipo de sustancias "no dependa de otra causa superior". Implica, en cambio, que "tiene una relación sempiterna con su causa primera" (lectio 25. Cursiva en el original).

El comentario a la proposición 25 revela, por eso, que si bien según Tomás de Aquino las nociones "esencia" (quidditas) y "forma" pueden tener sentidos equivalentes, ello no parece suceder cuando se trata de los significados de "esencia" y "ser" (esse). Precisamente, en el caso que analizamos "ser" se corresponde con la expresión "existir", es decir, refiere a que una sustancia recibe el ser de una causa primera que lo otorga por el modo de la creación.

Por otra parte, como el autor del Liber de causis establece en el título de la proposición 24 (25), en ella se examinan las sustancias inteligibles. Según Proclo, esas entidades eran aquellas que podían denominarse authypóstaton cuando cumplían con determinadas características: autosubsistentes, autárquicas y autosuficientes por participación de lo Uno/Bien (que es authypóstaton esencialmente), incorpóreas, automovidas y con la capacidad de autoconocimiento. Las notas que caracterizan a esas sustancias fueron ostensiblemente reducidas en el tratado de origen árabe y quedaron vinculadas casi exclusivamente a la operación intelectual de la inteligencia. Como hemos visto, las proposiciones 14 (15) y 24 (25) del De causis, refieren a ellas específicamente. La primera, enuncia que la operación de estas entidades consiste en el retorno hacia su esencia y ello garantiza el autoconocimiento y la segunda, indica que son causa de su propia esencia/forma, lo que las convierte en sustancias no sometidas a los procesos de generación y corrupción.

A modo de conclusión, nos interesa señalar que, si bien sobre la base de la lectura de los Elementos de teología, puede afirmarse que lo authypóstaton no solamente se predica de las inteligencias sino que también puede asignarse a las almas, en el tratado anónimo las proposiciones 14 (15) y 24 (25) refieren a las inteligencias. Aquino, en cambio, interpreta que la proposición 15 alude también al alma. Así, la reditio completa: 
Conviene al alma y a cualquier entidad que se conozca a sí misma porque toda sustancia de este tipo es simple, autosuficiente por sí misma como si no necesitara un sustento material.Y esa puede ser la tercera tesis, a saber, que el alma sea separable del cuerpo, como se expone en la proposición de Proclo (Expositio, lectio 15).

Como mencionamos más arriba, Tomás de Aquino cita las proposiciones 83 y 186 de los Elementos de teología en su Comentario al Libro de las causas. En la primera de ellas encuentra la conexión teórica entre la sustancia que se autoconstituye y su operación propia que es el autoconocimiento. En efecto, si el retorno se produce en relación con la operación (conocer) entonces debe también efectuarse en relación con la esencia pues la operación sigue a la esencia. Por otra parte, la proposición 186 de Proclo le otorga a Tomás la oportunidad de afirmar la autosubsistencia del alma y. consecuentemente, su capacidad para separarse del cuerpo.

En efecto, en el comentario a la proposición 15 del Liber de causis, el principio que enuncia la superioridad de la esencia sobre la operación resulta una justificación metafísica para sostener la inmortalidad del alma. Sin embargo, como intentamos mostrar, también produce un problema relativo a la noción de causalidad eficiente cuando se entiende como la "causa de la formación" de una sustancia o, en cambio, cuando significa dar el ser y, en ese sentido alude a la creación de una entidad. Como hemos visto, Tomás de Aquino resuelve este problema en su comentario a la proposición 25 ofreciendo una definición del concepto de "generación". Así, la capacidad para autoconstituirse que Proclo asignaba a las sustancias intelectuales (inteligencias y almas) es leída por el autor de la Expositio como una perfección que deviene, ya no de su capacidad para retornar hacia sí misma sino de la relación continua y sempiterna con la causa primera.

\section{BIBLIOGRAFÍA}

Acar, R. (2005), Talking about God and Talking about Creation (Leiden - Boston: Brill)

Aertsen, J. (1988), Nature and Creature Thomas Aquinas's Way of Thought (Leiden - New York: Brill).

Bertelloni, F. (2001), "La contribución de la filosofia a la formación del pensamiento político laico a fines del siglo XIII y comienzos del siglo XIV”, Mirabilia, 1: 190203.

Boese, H (1987) (ed.), Proclus. Elementatio Theologica, translata a Guillielmo de Moerbecca (Louvain: Louvain University Press).

D’Amico, C. (2008) (ed.), Todo y Nada de Todo. Selección de textos del neoplatonismo medieval (Buenos Aires: Winograd). 
D’Ancona, C. (1986), Tommaso D’Aquino Commento al Libro delle Cause (Milán: Rusconi).

D’Ancona, C. (1995), Recherches sur le Liber de Causis (Paris:Vrin).

D’Ancona, C. (2003), Plotino. La discesa dell'anima nei corpi (Padua: Il polígrafo)

D’Ancona, C. (2005), Storia della filosofía nell' islam medievale (Torino: Einaudi).

De Libera, A. (1990), "Albert le Grand et Thomas de Aquin interprètes du Liber de causis", Revue des Sciences philosophiques et theologiques, 74: 347-378.

Dillon, J. M. (2014), “Dionisio”, en S. Gersh (ed.), Interpreting Proclus: From Antiquity to the Renaissance (Cambridge: Cambridge University Press, 111-124).

Dodds, E. R. (1963), Proclus. The Elements of Theology. A Revised Text with Translation, Introduction and Commentary (Oxford: Clarendon Press).

Florido, L. (2005), “Translatio Studiorum: Traslado de los libros y diálogo de las civilizaciones en la Edad Media", Revista General de Información y Documentación, 15: 51-77.

Gersh, S. (2009), Da Giamblico a Eringena. Origini e sviluppi della tradizione pseudo-dionisiana (Bari: Di Pagina).

Gersh, S. (2014) (ed.), Interpreting Proclus. From Antiquity to the Renaissance (Cambridge: Cambridge University Press)

Imbach, R. (1978), “Le (néo-)platonisme medieval, Proclus latin et l'école dominicaine allemande", Revue de Théologie et de Philosophie, 110: 427-448

Klibansky, R. (1943), Plato's Parmenides in the Middle Ages and the Renaissance (London: Warburg Institute).

Ludueña, E. (2008), “El Pseudo Areopagita”, en C. D’Amico (ed.), Todo y Nada de Todo: Selección de textos del neoplatonismo medieval (Buenos Aires: Winograd, 75-86).

Saffrey, H.-D. (1954) (ed.), Tomae de Aquino, Expositio Super librum De causis (Friburgo: Société Philosophique).

Saffrey, H.-D. (1963), "L'État actuel des recherches sur le Liber de causis comme source de la métaphysique au Moyen Age", Die Metaphysik in Mittelater (Miscelanea Medievalia), 2: 267-281.

Scarpelli Cory, T. (2017), "Reditio completa, reditio incompleta: Aquinas and the Liber de causis prop. 15 on Reflexivity and Incorporeality" en A. Fidora y N. Polloni (2017) (eds.), Appropriation, Interpretation and Criticism: Philosophical and Theological Exchanges between the Arabic, Hebrew and Latin Intellectual Traditions, Barcelona Roma: FIDEM, 185-229).

Siorvanes, L. (1996), Proclus (New Haven - London:Yale University Press).

Steel, C. (2014), "William of Moerbeke, translator of Proclus" en S. Gersh (ed.), Interpreting Proclus: From Antiquity to the Renaissance (Cambridge: Cambridge University Press, 247-263).

Recibido: 02-06-2020; aceptado: 20-10-2020 